\title{
ON THE SET OF PERIODIC SOLUTIONS OF DIFFERENTIAL EQUATIONS OF RICCATI TYPE
}

\author{
by H. S. HASSAN
}

(Received 29th November 1983)

\section{Introduction}

The purpose of this paper is to expand upon the results obtained in [4].

We consider the set $H$ of differential equations

$$
\dot{z}=z^{2}+p(t) z+r(t) \quad(z \in \mathbb{C}, t \in \mathbb{R}),
$$

where $p$ and $r$ are continuous real-valued functions of period $\omega$ ( $\omega$ being fixed throughout). The equation (1.1) is denoted by $\mathrm{P}$ or $(p, r)$, and we regard $H$ as the set of pairs of continuous functions of period $\omega$. On $H$ we define a norm:

$$
\|P\|=\max \{|p(t)|, \quad|r(t)| ; \quad 0 \leqq t \leqq \omega\}
$$

then

$$
(H,\|\cdot\|) \text { is a Banach space. }
$$

We introduce some notation and recall some of the preparatory results from [2] and [4]. The solution of (1.1) satisfying $z\left(t_{0}\right)=c$ is written $z_{\mathrm{p}}\left(t ; t_{0}, c\right)$. The periodic solutions of (1.1) are determined by the zeros of

$$
q_{\mathrm{p}}: c \mapsto z_{\mathrm{P}}(\omega ; 0, c)-c .
$$

The domain of definition of $q_{\mathrm{P}}$ is an open set $Q_{\mathrm{P}} \subset \mathbb{C}$. We also define

$$
q: H \times \mathbb{C} \rightarrow \mathbb{C} ;(\mathrm{P}, c) \mapsto q_{\mathrm{P}}(c) .
$$

The domain of definition of $q$ is an open set $Q$ of $H \times \mathbb{C}$; on $Q, q$ is holomorphic in $c$ and continuous in $\mathrm{P}$. If $\mathrm{P}_{n} \rightarrow \mathrm{P}$ in $H, c_{n} \rightarrow c$ in $\mathbb{C}$, and $q\left(\mathrm{P}_{n}, c_{n}\right)=0$, then either $q(\mathrm{P}, c)=0$ or the solution $z_{\mathrm{P}}(t ; 0, c)$ is not defined for all $t \in[0, \omega]$.

The set of zeros of $q_{\mathrm{p}}$ is denoted by $B_{\mathrm{p}}$. In [2] and [4] the multiplicity of a periodic solution $\phi$ of (1.1) is defined as the multiplicity of $\phi(0)$ as a zero of $q_{\mathrm{p}}$. Also (1.1) is said to have a singular periodic solution if there are sequences $\left(P_{n}\right)$ and $\left.c_{n}\right)$ in $H$ and $\mathbb{C}$, respectively, such that $q\left(\mathrm{P}_{n}, c_{n}\right)=0$ but either $\mathrm{P}_{n} \rightarrow \mathrm{P}, c_{n} \rightarrow c$ and $z_{\mathrm{P}}(t ; 0, c)$ is not defined for $0 \leqq t \leqq \omega$, or $\mathrm{P}_{n} \rightarrow \mathrm{P}$ and $c_{n} \rightarrow \infty$. The set of $\mathrm{P} \in H$ with no singular periodic solutions is denoted by $\mathscr{A}$. We quote some results from [4]. 
Theorem 1.1 If $\mathrm{P} \notin \mathscr{A}$, then $\mathrm{P}$ has a solution which is unbounded both as $t$ increases and as $t$ decreases, and is defined for a t-interval of length less than $\omega$.

It was shown in [2] that $\mathscr{A}$ is an open set: by a component of $\mathscr{A}$ we mean a maximal connected subset of $\mathscr{A}$.

Theorem 1.2. If $\mathrm{P}_{1}$ and $\mathrm{P}_{2}$ are in the same component of $\mathscr{A}$, they have the same number of periodic solutions.

Remark. In Theorem 1.2 the multiplicity of solutions is taken into account. This we do throughout the paper.

It is shown in [4] that a member of $H$ either has no periodic solutions, two periodic solutions or infinitely many. We make the following definition.

Definition 1.3. $\quad H_{1}=\{\mathrm{P} ; \mathrm{P}$ has exactly two periodic solutions, both real $\}$, $H_{2}=\{\mathrm{P} ; \mathrm{P}$ has exactly two periodic solutions neither real $\}$,

$H_{3}=\{\mathrm{P} ;$ every non-real solution is periodic; no real solution is periodic $\}$, $H_{4}=\{\mathrm{P} ; \mathrm{P}$ has no periodic solution $\}$.

The results summarised in the following theorems were also proved in [4].

Theorem 1.4. $H$ is the disjoint union of $H_{1}, H_{2}, H_{3}$ and $H_{4}$.

Theorem 1.5. (1) $H_{1} \cup H_{2}$ is a component of $\mathscr{A}$,

(2) $H_{4}$ contains infinitely many components of $\mathscr{A}$.

(3) $H_{3} \cap \mathscr{A}=\varnothing$.

In [2] equation (1.1) was investigated by considering the related linear equation $\mathrm{P}^{*}$ :

$$
\ddot{u}-p(t) \dot{u}+r(t) u=0
$$

Since we shall also use this technique, we briefly describe the necessary background.

Equation (1.2) is obtained from (1.1) by the transformation $z=-\dot{u} u^{-1}$. Since $u \equiv 0$ is a solution of $P^{*}$, a solution of $P^{*}$ cannot vanish together with its derivative; consequently, every non-trivial solution of $\mathrm{P}^{*}$ yields a solution of $\mathrm{P}=(p, r)$. Conversely every solution of $P$ can be written as $-\dot{u} u^{-1}$, where $u$ is a solution of $P^{*}$. The period solutions of $P$ can be studied by choosing a suitable base for the solutions of $P^{*}$.

We take a Floquet base $\left(u_{1}, u_{2}\right)$ for $P^{*}$ and use this to examine the periodic solutions of $P$. A Floquet base is either of the form

$$
\left(\alpha_{1}(t) e^{\lambda_{1} t}, \alpha_{2}(t) e^{\lambda_{2} t}\right)
$$

or

$$
\left(\alpha_{1}(t) \mathrm{e}^{\lambda_{1} t},\left(t \alpha_{1}(t)+\alpha_{2}(t)\right) e^{\lambda_{1} t}\right)
$$

where $\lambda_{1}, \lambda_{2} \in \mathbb{C}$ and $\alpha_{1}, \alpha_{2}: \mathbb{R} \mapsto \mathbb{C}$ are $\omega$-periodic. (For details of the theory leading to the existence of such basis see Coddington and Levinson [1]). 
We can so choose $u_{1}$ and $u_{2}$ that either both are real or $u_{1}$ and $u_{2}$ have independent real and imaginary parts and $u_{1}=\vec{u}_{2}$. The form of the basis depends only on the nature of the characteristic multipliers. It is always of the first form except when $P^{*}$ has equal multipliers (necessarily real).

We adopt the convention that

$$
-\frac{\pi}{\omega}<\operatorname{Im} \lambda_{1}, \operatorname{Im} \lambda_{2} \leqq \pi / \omega
$$

We shall need formulae for $p, r$ in terms of a base $\left(u_{1}, u_{2}\right)$ of $\mathrm{P}^{*}$ :

$$
\begin{aligned}
& p(t)=\frac{\ddot{u}_{1} u_{2}-\ddot{u}_{2} u_{1}}{\dot{u}_{1} u_{2}-\dot{u}_{2} u_{1}}, \\
& r(t)=\frac{\ddot{u}_{1} \dot{u}_{2}-\ddot{u}_{2} \dot{u}_{1}}{\dot{u}_{1} u_{2}-\dot{u}_{2} u_{1}} .
\end{aligned}
$$

This paper is concerned with the topological properties of $H_{i}(i=1,2,3,4)$. It will be shown that the boundary between $H_{1}$ and $H_{2}$ is a manifold and $H_{3} \subset \bar{H}_{4} \backslash H_{4}$.

I wish to express my gratitude to $\mathrm{Dr} \mathrm{N}$. G. Lloyd for his valuable guidance and encouragement during the preparation of this paper.

\section{Two periodic solutions}

In this section we shall study some of the properties of $H_{1}$ and $H_{2}$.

In [2], Lloyd proved the following result which we quote without proof.

Theorem 2.1. If $r(t)<0$ for all $t$, then $\mathrm{P}$ has exactly two $\omega$-periodic solutions, counting multiplicity.

Theorem 2.1 was proved by showing that an equation with $r<0$ is in the component of the origin in the set $\mathscr{A}$ of equations with no singular periodic solution. From this it follows that $(p, r)$ must have the same number of periodic solutions as the equation $\dot{x}=x^{2}$ which is two.

Theorem 2.1 simply states that, if $r(t)<0$ for all $t$, then $\mathrm{P} \in H_{1} \cup H_{2}$. We shall prove that if $\mathrm{P}$ satisfies the hypothesis of Theorem 2.1 , then it does not belong to $\mathrm{H}_{2}$. In order to prove this result we need to recall that if $x(t)$ is a non-real solution of $\mathrm{P}$, then there exist differentiable real-valued functions $s(t)$ and $\phi(t)$ such that $x(t)=s(t) e^{i \phi(t)}$ and consequently

$$
\begin{gathered}
\dot{s}(t)=\left(s^{2}(t)+r(t)\right) \cos \phi(t)+s(t) p(t), \\
s(t) \phi(t)=\left(s^{2}(t)-r(t)\right) \sin \phi(t)
\end{gathered}
$$

for all $t$. 
Theorem 2.2 If $p$ and $r$ not both constants and $r<0$ for all $t$, then $\mathrm{P}=(p, r) \in H_{1}$.

Proof. Suppose, if possible, that for some $\xi \in \mathbb{C}$, where $\operatorname{Im} \xi \neq 0, x_{\mathrm{p}}(t ; 0, \xi) \equiv s(t) e^{i \phi(t)}$ is $\omega$-periodic. So that $x_{\mathrm{p}}(t ; 0, \xi),(0 \leqq t \leqq \omega)$, forms a closed curve either in the upper halfplane or in the lower half-plane. Hence there exists $t_{0} \in[0, \omega]$ such that $\phi\left(t_{0}\right)=0$.

Since $r(t)<0$ and $\sin \phi(t) \neq 0,(2.2)$ gives $\phi(t) \neq 0$ for all $t$, which contradicts the existence of $t_{0}$.

We see in the next lemma that if $x(t)$ is a real $\omega$-periodic solution, then

$$
\|x\|=\sup _{0 \leqq t \leqq \omega}|x(t)|
$$

depends only on $\|P\|$.

Lemma 2.3. Suppose that $x(t)$ is a real $\omega$-periodic solution of $P$. Then

$$
\|x\| \leqq\|P\|+\sqrt{\|P\|^{2}+2\|P\|}
$$

Proof. Suppose, if possible, that $x(t)$ is a real $\omega$-periodic solution of $P$ and

$$
\left|x\left(t_{0}\right)\right|>\|\mathbf{P}\|+\sqrt{\|\mathbf{P}\|^{2}+2 \mid\|\mathbf{P}\|}
$$

for some $t_{0} \in[0, \omega]$. We have two cases to consider: (i) $x\left(t_{0}\right)>0$ and (ii) $x\left(t_{0}\right)<0$.

Case (i). Suppose that $x\left(t_{0}\right)>0$. Since $x(t)$ is differentiable and periodic, there exists $t_{1} \in[0, \omega]$ such that $x\left(t_{1}\right)=\max _{0 \leqq t \leqq \omega} x(t)$. Hence $\dot{x}\left(t_{1}\right)=0$ and moreover

$$
x\left(t_{1}\right)>\|\mathrm{P}\|+\sqrt{\|\mathrm{P}\|^{2}+2\|\mathrm{P}\|} .
$$

But $x(t)$ is a solution of equation $P$, so for all $t$

$$
\dot{x}(t)=x^{2}(t)+p(t) x(t)+r(t) \geqq x^{2}(t)-\|\mathrm{P}\|(x(t)+1) .
$$

It is easily seen that

$$
x^{2}\left(t_{1}\right)>\|\mathrm{P}\|\left(x\left(t_{1}\right)+1\right)
$$

hence,

$$
\dot{x}\left(t_{1}\right)>0, \text { a contradiction. }
$$

Case (ii). Suppose that $x\left(t_{0}\right)<0$. Consider the transformations $t \rightarrow-t, x \rightarrow-x$, then $-x(t)$ is a periodic solution of

$$
\dot{x}=x^{2}-p(t) x+r(t)
$$

Hence we have case (i) and the lemma is proved. 
Theorem 2.4. $H_{1}$ is a closed subset of $H$.

Proof. Suppose that $\left(\mathrm{P}_{n}\right)$ is a convergent sequence in $H_{1}$ and $\mathrm{P}_{n} \rightarrow \mathrm{P}$ as $n \rightarrow \infty$. Suppose, if possible, that $\mathrm{P} \notin H_{1}$; that is, $B_{\mathrm{P}}$ has no real members. Let $M_{n}=\left\|\mathrm{P}_{n}\right\|$. Since $\mathrm{P}_{n} \rightarrow \mathrm{P}$ as $n \rightarrow \infty,\left\{M_{n}: n \in \mathbb{Z}^{+}\right\}$is bounded. Let $M^{*}=\sup _{n} M_{n}$. Choose $c_{n} \in B_{\mathrm{P}_{n}}(n=1,2, \ldots)$. By Lemma 2.3,

$$
\left|x_{P_{n}}\left(t ; 0, c_{n}\right)\right|<M^{*}+\sqrt{\left(M^{*}\right)^{2}+2 M^{*}}, \quad(0 \leqq t \leqq \omega) .
$$

Hence we can assume, without loss of generality, that $c_{n} \rightarrow c_{0}$ as $n \rightarrow \infty$ for some $c_{0} \in \mathbb{R}$. So either $q\left(\mathrm{P}, c_{0}\right)=0$ or $x_{\mathrm{P}}\left(t ; 0, c_{0}\right)$ is not defined in $[0, \omega]$. Since, by hypothesis, $B_{\mathrm{P}}$ contains no real member, $q\left(P, c_{0}\right)=0$ is excluded. Hence there exists $\tau \in(0, \omega]$ such that $\left|x_{\mathrm{P}}\left(t ; 0, c_{0}\right)\right| \rightarrow \infty$ as $t \uparrow \tau$. But $x_{\mathrm{P}_{n}}\left(t ; 0, c_{n}\right) \rightarrow x_{\mathrm{P}}\left(t ; 0, c_{0}\right)$ as $n \rightarrow \infty$ for all $t \in[0, \omega)$. It follows that (2.4) is satisfied for $x_{\mathrm{P}}\left(t ; 0, c_{0}\right)$, a contradiction. Therefore $q\left(\mathrm{P}, c_{0}\right)=0$, hence $\mathrm{P} \in H_{1}$ and the theorem is proved.

Corollary 2.5. If $p$ and $r$ are not constant and $r(t) \leqq 0$ for $0 \leqq t \leqq \omega$, then $\mathrm{P} \in H_{1}$.

Proof. Let $\mathrm{P}_{n}=\left(p, r_{n}\right)$ where $r_{n}=r-\frac{1}{n}(n=1,2, \ldots)$. Hence, by Theorem 2.2 , for all $n$ $\mathrm{P}_{n} \in H_{1}$ and $\mathrm{P}_{n} \rightarrow \mathrm{P}$ as $n \rightarrow \infty$. By Theorem 2.4, $\mathrm{P} \in H_{1}$. Whence the result is proved.

Corollary 2.6. $\mathrm{H}_{2}$ is open in $\mathrm{H}$.

Proof. We know from [4] that $H_{1} \cup H_{2}$ is open in $H$ and we have proved that $H_{1}$ is closed in $H$. Since $H_{1} \cap H_{2}$ is empty, $H_{2}$ is open in $H$ and the corollary is proved.

Directly from Corollary 2.5 , we have if $\mathrm{P}_{1}=(p, 0)$, then $\mathrm{P}_{1} \in H_{1}$. We shall use this result to show that under certain conditions $\mathrm{P} \in H_{1}(\mathrm{P}=(p, r))$ if $|r|$ is small enough, irrespective of the size of $P$.

The following lemma can be deduced from Proposition 3.1 of Lloyd [2].

Lemma 2.7. If $\int_{0}^{\omega} p(t) d t \neq 0$, then $x=0$ is an $\omega$-periodic solution of $\mathbf{P}=(p, 0)$ with multiplicity 1.

Theorem 2.8. If $\int_{0}^{\infty} p(t) d t \neq 0$, and $|r|$ is small enough, then $\mathrm{P} \in H_{1}$.

Proof. Let $\mathrm{P}_{1}=(p, 0)$. By Corollary $2.5, \mathrm{P}_{1} \in H_{1}$. By Lemma 2.7 the zero solution is a periodic solution of $\mathrm{P}_{1}$ of multiplicity 1 ; there is therefore another periodic solution $x_{\mathrm{P}}(t ; 0, c)$, say, with $c$ real and non-zero. We know that $H_{1} \cup H_{2}$ is open. Suppose then, if possible, that there exists a sequence $\left(\mathrm{P}_{n}\right)$ in $H_{2}$ convergent to $\mathrm{P}_{1}$. Let $c_{1}^{n}, c_{2}^{n}$ be the two starting points of the two $\omega$-periodic solutions of $P_{n}$. Then, $c_{1}^{n} \rightarrow 0$ and $c_{2}^{n} \rightarrow c$ as $n \rightarrow \infty$. But $c_{1}^{n}, c_{2}^{n}$ are complex conjugates; it follows that $c=0$, a contradiction. Therefore, $\mathrm{P}_{1} \in$ int $H_{1}$ and the theorem is proved.

\section{The boundary between $\mathrm{H}_{1}$ and $\mathrm{H}_{2}$}

In this section we study the characteristics of the boundary between $H_{1}$ and $H_{2}$ and show that it is a manifold. 
Definition. Let $H_{11}=\left\{\mathrm{P} ; B_{\mathrm{P}}\right.$ contains exactly one point $\}$.

Remark. It is clear that

$$
H_{11} \cap H_{2}=H_{11} \cap H_{3}=H_{11} \cap H_{4}=\varnothing \text {. }
$$

So $H_{11} \subset H_{1}$ and if $x \in B_{\mathrm{P}}\left(\mathrm{P} \in H_{11}\right)$, then $i(q(\mathrm{P}), x, 0)=$,2 , (where $i(q(\mathrm{P},), x, 0$.$) is the index$ of $q(\mathrm{P},$.$) at the 0$-point $x$, for more details sec [5]).

We prove that $H_{11}$ is the boundary between $H_{1}$ and $H_{2}$.

Theorem 3.1. $\quad H_{1}$ is a perfect subset of $H$.

Proof. Let $\mathrm{P}=(p, r) \in H_{1}$. Suppose that $\phi$ is one of the $\omega$-periodic solutions of $\mathrm{P}$. It can be checked that $\phi_{n}=\phi+1 / n\left(n \in \mathbb{Z}^{+}\right)$is a real $\omega$-periodic solution of

$$
\mathrm{P}_{n}=\left(p-\frac{2}{n}, r-\frac{p}{n}+\frac{1}{n^{2}}\right),
$$

and $\mathrm{P}_{n} \rightarrow \mathrm{P}$ as $n \rightarrow \infty$. Since $B_{\mathrm{P}_{n}}$ contains at least one real element, namely $\phi_{n}(0), \mathrm{P}_{n} \in H_{1}$. Hence $\mathrm{P}$ is an accumulation point of $H_{1}$. Since $H_{1}$ is closed, $H_{1}$ is perfect and the result is proved.

Recall that $P^{*}$ was the related linear equation (1.2).

Lemma 3.2. If $\mathrm{P} \in H_{11}$, then the Floquet base of $\mathrm{P}^{*}$ is of form

$$
\left(e^{\lambda t} \alpha_{1}(t), e^{\lambda t}\left(t \alpha_{1}(t)+\alpha_{2}(t)\right)\right.
$$

Proof. Suppose that $\left(e^{\lambda_{1} t} \alpha_{1}(t), e^{\lambda_{2} t} \alpha_{2}(t)\right)$ is a base of $\mathrm{P}^{*}$. Hence if

$$
u(t)=c_{1} e^{\lambda_{1} t} \alpha_{1}(t)+c_{2} e^{\lambda_{2} t} \alpha_{2}(t) \quad\left(c_{1}, c_{2} \in \mathbb{C}\right),
$$

then

$$
\phi(t)=-\frac{c_{1} e^{\lambda_{1} t} \beta_{1}(t)+c_{2} e^{\lambda_{2} t} \beta_{2}(t)}{c_{1} e^{\lambda_{1} t} \alpha_{1}(t)+c_{2} e^{\lambda_{2} t} \alpha_{2}(t)}
$$

is a solution of $\mathrm{P}$, where $\beta_{i}=\dot{\alpha}_{i}+\lambda_{i} \alpha_{i}(i=1,2)$.

Hence $\phi$ is $\omega$-periodic if and only if

$$
c_{1} c_{2}\left(e^{\lambda_{1} \omega}-e^{\lambda_{2} \omega}\right)=0
$$

and $c_{1} e^{\lambda_{1} t} \alpha_{1}(t)+c_{2} e^{\lambda_{2} t} \alpha_{2}(t)$ does not vanish.

We have one of the following two cases:

(i) $\lambda_{1} \neq \lambda_{2}$,

(ii) $\lambda_{1}=\lambda_{2}$. 
Case (i). By (3.2) $\phi$ is $\omega$-periodic if and only if $c_{1}=0$ or $c_{2}=0$. So, by (3.1), $\mathrm{P}$ has only two $\omega$-periodic solutions, namely

$$
\phi_{1}(t)=-\frac{\beta_{1}(t)}{\alpha_{1}(t)} \quad \text { and } \quad \phi_{2}(t)=-\frac{\beta_{2}(t)}{\alpha_{2}(t)}
$$

provided that $\alpha_{1}(t), \alpha_{2}(t) \neq 0$. Since $u_{1}=e^{\lambda_{1} t} \alpha_{1}(t)$ and $u_{2}=e^{\lambda_{2} t} \alpha_{2}(t)$ are linearly independent, $\phi_{1} \neq \phi_{2}$. If both $u_{1}$ and $u_{2}$ are real, then their zeros, according to the Sturm separation theorem, interlace; both $\phi_{1}$ and $\phi_{2}$ are then defined for all $t$ or neither is. Hence if $P$ has a real $\omega$-periodic solution, then it has two distinct such solutions; so $\mathrm{P} \notin H_{11}$.

Case (ii). By (3.2) every solution of $P$ is either $\omega$-periodic or is defined for a time less than $\omega$. Hence if $P$ has an $\omega$-periodic solution, then it has infinitely many. Hence $\mathrm{P} \notin H_{11}$.

We conclude that the base of $P^{*}$ is of the form

$$
\left(e^{\lambda t} \alpha_{1}(t), e^{\lambda t}\left(t \alpha_{1}(t)+\alpha_{2}(t)\right)\right)
$$

Remark. We note that $\lambda_{1}-\lambda_{2} \neq 2 n \pi i / \omega$ for all $n \in \mathbb{Z}^{+}$, (because $\left|\operatorname{Im} \lambda_{1}\right|<\pi / \omega$ and $\left.\left|\operatorname{Im} \lambda_{2}\right| \leqq \pi / \omega\right)$.

Theorem 3.3. $H_{11} \subset \partial H_{2}$.

Proof. Suppose that $\mathrm{P}=(p, r) \in H_{11}$. Then by Lemma $3.2 \mathrm{P}^{*}$ has a base $u_{1}=e^{\lambda t} \alpha_{1}(t)$, $u_{2}=e^{\lambda t}\left(t \alpha_{1}(t)+\alpha_{2}(t)\right)$. Since $u_{1}, u_{2}$ are independent solutions of $P^{*}$, computing the Wronskian gives

$$
\dot{\alpha}_{1}(t) \alpha_{2}(t)-\alpha_{1}(t) \dot{\alpha}_{2}(t)-\alpha_{1}^{2}(t) \neq 0
$$

for all $t$.

Substituting $u_{1}$ and $u_{2}$ in (1.3) and (1.4) gives us

$$
\begin{aligned}
& p=2 \lambda+\frac{\ddot{\alpha}_{1} \alpha_{2}-\alpha_{1} \ddot{\alpha}_{2}-2 \alpha_{1} \dot{\alpha}_{2}}{\dot{\alpha}_{1} \alpha_{2}-\alpha_{1} \dot{\alpha}_{2}-\alpha_{1}^{2}} \\
& r=\lambda^{2}+\lambda(p-2 \lambda)-\frac{\dot{\alpha}_{1} \ddot{\alpha}_{2}-\ddot{\alpha}_{1} \alpha_{2}-\alpha_{1} \ddot{\alpha}_{2}+2 \dot{\alpha}_{1}^{2}}{\dot{\alpha}_{1} \alpha_{2}-\alpha_{1} \dot{\alpha}_{2}-\alpha_{1}^{2}} .
\end{aligned}
$$

We construct a sequence $\mathrm{P}_{n}$ in $H_{2}$ converging to $\mathrm{P}$.

For $n \in \mathbb{Z}^{+}$let

$$
V_{n}=\left(\alpha_{1}+\frac{\mathrm{i}}{n} \alpha_{2}\right) \exp \left(\lambda+\frac{i}{n}\right) t
$$


and

$$
W_{n}=\bar{V}_{n}
$$

Substituting $V_{n}$ and $W_{n}$ in (1.3) and (1.4) gives us

$$
p_{n}=2 \lambda+\frac{\alpha_{1} \ddot{\alpha}_{2}-\ddot{\alpha}_{1} \alpha_{2}+2 \alpha_{1} \dot{\alpha}_{1}+2 \alpha_{2} \dot{\alpha}_{2} / n^{2}}{\alpha_{1} \dot{\alpha}_{2}-\dot{\alpha}_{1} \alpha_{2}+\alpha_{1}^{2}+\alpha_{2}^{2} / n^{2}}
$$

and

$$
r_{n}=\frac{\dot{\alpha}_{1} \ddot{\alpha}_{2}-\ddot{\alpha}_{1} \dot{\alpha}_{2}+2 \dot{\alpha}_{1}-\alpha_{1} \ddot{\alpha}_{1}-\lambda\left(\alpha_{1} \ddot{\alpha}_{2}+\ddot{\alpha}_{1} \alpha_{2}+2 \alpha_{1} \dot{\alpha}_{1}\right)+\lambda^{2}\left(\alpha_{1} \dot{\alpha}_{1}-\dot{\alpha}_{1} \alpha_{2}+\alpha_{1}\right)+E / n^{2}+\alpha_{2}^{2} / n^{4}}{\alpha_{1} \dot{\alpha}_{2}-\dot{\alpha}_{1} \alpha_{2}+\alpha_{1}^{2}+\alpha_{2}^{2} / n^{2}}
$$

where $E=\left(\dot{\alpha}_{2}+\alpha_{1}+\lambda \alpha_{2}\right)\left(\alpha_{1}+2 \dot{\alpha}_{2}+2 \lambda \alpha_{2}\right)-\alpha_{2}\left(\dot{\alpha}_{1}+\lambda \alpha_{1}\right)$

$$
-\alpha_{2}\left(\ddot{\alpha}_{2}+2 \dot{\alpha}_{1}+2 \lambda\left(\alpha_{1}+\dot{\alpha}_{2}\right)+\lambda^{2} \alpha_{2}\right) \text {. }
$$

It can be checked that $\mathrm{P}_{n}=\left(p_{n}, r_{n}\right) \in H$ and $\left(V_{n}, W_{n}\right)$ is a base of $\mathrm{P}_{n}^{*}$. Since for large $n$, $(\lambda+i / n) \neq(\lambda-i / n)$ then $\mathrm{P}_{n} \in H_{1} \cup H_{2}$. It can be checked that

$$
\phi_{n}=-\left[\lambda+\frac{i}{n}+\left(\dot{\alpha}_{1}+\frac{i}{n} \dot{\alpha}_{2}\right) /\left(\alpha_{1}+\frac{i}{n} \alpha_{2}\right)\right]
$$

is an $\omega$-periodic solution of $P_{n}$. Therefore for large $n, P_{n} \in H_{2}$. Since $P_{n} \rightarrow P$ as $n \rightarrow \infty$, $\mathrm{P} \in \partial \mathrm{H}_{2}$ and the theorem is proved.

Theorem 3.4 $H_{11}$ is the boundary "between" $H_{1}$ and $H_{2}$; that is, $H_{11}=\bar{H}_{1} \cap \bar{H}_{2}$.

Proof. Suppose that $\mathrm{P} \in \bar{H}_{1} \cap \bar{H}_{2}$. Since $H_{1}$ is a closed subset of $H, \mathrm{P} \in H_{1}$. Suppose that $\mathrm{P} \notin H_{11}$; then $B_{\mathrm{P}}$ contains exactly two real elements, $x_{1}, x_{2}$ say. Since $\mathrm{P} \in \partial H_{2}$ there exists a sequence $\left(\mathrm{P}_{n}\right)$ in $H_{2}$ convergent to $\mathrm{P}$. If $c_{n} \in B_{\mathrm{P}_{n}}$, then $\bar{c}_{n} \in B_{\mathrm{P}_{n}}$. Hence we can assume without loss of generality that $c_{n} \rightarrow x_{1}$ and $\bar{c}_{n} \rightarrow x_{2}$ as $n \rightarrow \infty$. But $x_{1} \neq x_{2}$ are real, a contradiction. Therefore $\mathrm{P} \in H_{11}$.

Conversely, if $\mathrm{P} \in H_{11}$, then by Theorem 3.3 $\mathrm{P} \in \bar{H}_{2}$ and Theorem $3.1 \mathrm{P} \in \bar{H}_{1}$ and the theorem is proved.

Lloyd in [2] proved the following result which we quote without proof.

(Recall that an $\omega$-periodic solution $\phi$ is simple if it has multiplicity 1 ).

Lemma 3.5. Let $\mathrm{P}=(p, r) \in H_{1}$ and suppose that $\phi$ is $\omega$-periodic solution of $\mathrm{P}$. Then $\mathrm{P} \in \mathrm{H}_{11}$ if and only if

$$
\int_{0}^{\infty}(2 \phi(t)+p(t)) d t=0
$$

Let $H^{1}=\mathbb{P}^{1} \times \mathbb{P}^{1}$, where $\mathbb{P}^{1}$ is the set of all differentiable functions in $\mathbb{P}$, and $\mathbb{P}$ is the set of all functions $P: \mathbb{R} \rightarrow \mathbb{B}$ continuous and $\omega$-periodic, $H^{1}$ is a subspace of $H$. On $H^{1}$ 
define the norm

$$
\left\|\left(r_{1}, r_{2}\right)\right\|_{1}=\max _{0 \leqq t \leqq \omega}\left(\left|r_{1}(t)\right|,\left|r_{2}(t)\right|,\left|\dot{r}_{2}(t)\right|\right)
$$

Note that this norm is not the norm induced by the norm on $H$.

Let

$$
H^{11}=\left\{\left(\alpha_{1}, \alpha_{2}\right) \in H^{1} ; \int_{0}^{\omega}\left(\alpha_{1}(t)+2 \alpha_{2}(t)\right) d t=0\right\}
$$

Lemma 3.6. $\quad H^{11}$ is a hyperplane in $H^{1}$.

Proof. Let $\mathscr{T}: H^{1} \rightarrow \mathbb{R}$ be defined by

$$
\mathscr{T}\left(\alpha_{11}, \alpha_{2}\right)=\int_{0}^{\infty}\left(\alpha_{1}(t)+2 \alpha_{2}(t)\right) d t
$$

It is clear that $\mathscr{T}$ is a non-zero linear functional and

$$
H^{11}=\left\{\left(\alpha_{1}, \alpha_{2}\right) \in H^{1} ; \quad \mathscr{T}\left(\alpha_{1}, \alpha_{2}\right)=0\right\} .
$$

Hence $H^{11}$ is a hyperplane.

Theorem 3.7. $\quad H_{11}$ is a manifold (modelled on a Banach space).

Proof. Let $L: H_{11} \rightarrow H^{11}$ be defined by

$$
L(p, r)=(p, \phi)
$$

where $\phi$ is the unique $\omega$-periodic solution of $(p, r)$. It is clear that $L$ is bijective, for if $(p, \phi) \in H^{11}$, let $r=\phi-\phi^{2}-p \phi$. Then by Lemma $3.5(p, r) \in H_{11}$ and $L(p, r)=(p, \phi)$, and if $L(p, r)=L\left(p^{\prime}, r^{\prime}\right)=(r, \phi)$, then $p^{\prime}=p$ and $r=\phi-\phi^{2}-p \phi=r^{\prime}$.

We shall prove that $L$ is continuous. If $\varepsilon>0$ is given, then there exists $\delta_{1}>0$ with $0<\delta_{1}<\varepsilon$ such that if

$$
(p, r),\left(p^{\prime}, r^{\prime}\right) \in H_{11} \quad \text { and } \quad\left\|\left(p-p^{\prime}, r-r\right)\right\|<\delta \text {, }
$$

then

$$
\max _{0 \leqq t \leqq \omega}\left|\phi(t)-\phi_{1}(t)\right|<\delta
$$

implies

$$
\max _{0 \leqq t \leqq \omega}\left|\phi^{2}(t)+p(t) \phi(t)+r(t)-\phi_{1}^{2}(t)-p^{\prime}(t) \phi_{1}(t)-r^{\prime}(t)\right| \leqq \varepsilon
$$

$\left(\phi, \phi_{1}\right.$ are the unique periodic solutions of $(p, r),\left(p^{\prime}, r^{\prime}\right)$ respectively). 
By Theorem 2 in [3] there exists $\delta_{2}$ with $0<\delta_{2}<\delta_{1}$ such that if $(p, r),\left(p^{\prime}, r^{\prime}\right) \in H_{11}$ and $\left\|\left(p-p^{\prime}, r-r^{\prime}\right)\right\|<\delta_{2}$, then

$$
\max _{0 \leqq t \leqq \omega}\left|\phi(t)-\phi_{1}(t)\right|<\delta_{1}
$$

Hence by (3.3) and (3.4),

$$
\max _{0 \leqq t \leqq \omega}\left|\phi(t)-\phi_{1}(t)\right|<\varepsilon
$$

for all $(p, r),\left(p^{\prime}, r^{\prime}\right) \in H_{11}$ for which $\left\|\left(p-p^{\prime}, r-r^{\prime}\right)\right\|<\delta_{2}$. Therefore by (3.5) and (3.4),

$$
\begin{aligned}
\left\|L(p, r)-L\left(p^{\prime}, r^{\prime}\right)\right\|_{1} & =\left\|(p, \phi)-\left(p^{\prime}, \phi_{1}\right)\right\|_{1} \\
& =\max _{0 \leqq t \leqq \omega}\left(\left|p(t)-p^{\prime}(t)\right|,\left|\phi(t)-\phi_{1}(t)\right|,\right. \\
& \left.\left|\phi(t)-\phi_{1}(t)\right|\right) \\
& <\varepsilon
\end{aligned}
$$

for all $(p, r),\left(p^{\prime}, r^{\prime}\right) \in H_{11}$, for which

$$
\left\|\left(p-p^{\prime}, r-r^{\prime}\right)\right\|<\delta_{2}
$$

Next we show that $L^{-1}$ is also continuous. For if $\varepsilon>0$ is given, then there exists $\delta$ such that $0<\delta<\varepsilon$ and if $(p, \phi),\left(p^{\prime}, \phi_{1}\right) \in H^{11}$ and $\left\|\left(p-p^{\prime}, \phi-\phi_{1}\right)\right\|_{1}<\delta$, then

$$
\begin{aligned}
\max _{0 \leqq t \leqq \omega}\left|r(t)-r^{\prime}(t)\right| & \leqq \max _{0 \leqq t \leqq \omega}\left|\phi(t)-\phi^{2}(t)-p(t) \phi(t)-\phi_{1}(t)+\phi_{1}^{2}(t)+p^{\prime}(t) \phi_{1}(t)\right| \\
& <\varepsilon
\end{aligned}
$$

Therefore,

$$
\begin{aligned}
/ L^{-1}(p, \phi)-L^{-1}\left(p^{\prime}, \phi_{1}\right) \| & =\left\|\left(p-p^{\prime}, r-r^{\prime}\right)\right\| \\
& \leqq \max _{0 \leqq t \leqq \omega}\left(\left|p(t)-p^{\prime}(t)\right|,\left|r(t)-r^{\prime}(t)\right|\right) \\
& <\varepsilon
\end{aligned}
$$

for all $(p, \phi),\left(p^{\prime}, \phi_{1}\right) \in H^{11}$ for which

$$
\left\|\left(p-p^{\prime}, \phi-\phi^{\prime}\right)\right\|<\delta .
$$

Hence $L$ is a homeomorphism and is bijective. Since $H^{11}$ is a hyperplane, $H_{11}$ is a manifold and the theorem is proved. 


\section{4. $\mathrm{H}_{3} \cup \mathrm{H}_{4}$ as a subset of $\mathrm{H}$}

In this section we study some of the properties of $H_{3} \cup H_{4}$. Recall that $\mathrm{P}^{*}$ denotes the equation

where $P=(p, r)$.

$$
\ddot{u}-p \dot{u}+r u=0,
$$

Lemma 4.1. If $\left(\alpha_{1}(t) e^{\lambda_{1} t}, \alpha_{2}(t) e^{\lambda_{2} t}\right)$ is a base of $\mathrm{P}^{*}$ and $\lambda_{1} \neq \lambda_{2}$, then $\mathrm{P} \notin H_{3}$.

Proof. It can be checked that the solutions of $P$ are

$$
\frac{-c_{1}\left(\dot{\alpha}_{1}+\lambda_{1} \alpha_{1}\right) e^{\lambda_{1} t}+c_{2}\left(\dot{\alpha}_{2}+\lambda_{2} \alpha_{2}\right) e^{\lambda_{2} t}}{c_{1} \alpha_{1} e^{\lambda_{1} t}+c_{2} \alpha_{2} e^{\lambda_{2} t}}
$$

where $c_{1}$ and $c_{2}$ are complex constants. Since $\alpha_{1} e^{\lambda_{1} t}$ and $\alpha_{2} e^{\lambda_{2} t}$ are linearly independent, the solution (4.1) is $\omega$-periodic if and only if

$$
c_{1} c_{2}\left(e^{\lambda_{1} \omega}-e^{\lambda_{2} \omega}\right)=0
$$

Since $\lambda_{1} \neq \lambda_{2}$ and $\left|\operatorname{Im} \lambda_{i}\right| \leqq \pi / \omega(i=1,2)$, we see from (4.1) and (4.2) that $\mathrm{P}$ has at most two $\omega$-periodic solutions, namely

$$
\frac{-\dot{\alpha}_{2}+\lambda_{2} \alpha_{2}}{\alpha_{2}} \text { and } \frac{-\dot{\alpha}_{1}+\lambda_{1} \alpha_{1}}{\alpha_{1}} .
$$

Therefore $\mathrm{P} \notin H_{3}$ and the lemma is proved.

Now suppose that $\left(\alpha_{1}(t) e^{\lambda_{1} t}, \alpha_{2}(t) e^{\lambda_{2} t}\right)$ is a base of $P^{*}$ and $\lambda_{1} \neq \lambda_{2}$. Then by Lemma 4.1 $\mathrm{P} \notin H_{3}$. It can be checked that

$$
\frac{-\dot{\alpha}_{1}+\lambda_{1} \alpha_{1}}{\alpha_{1}}, \text { and } \frac{-\dot{\alpha}_{2}+\lambda_{2} \alpha_{2}}{\alpha_{2}}
$$

are $\omega$-periodic solutions of $P$, provided $\alpha_{1}(t)$ and $\alpha_{2}(t)$ do not vanish. Hence we have proved the following lemma.

Lemma 4.2. Let $\alpha_{i}, \lambda_{i}(i=1,2)$ and $\mathrm{P}$ be as in Lemma 4.1. If $\alpha_{1}(t) \neq 0$ or $\alpha_{2}(t) \neq 0$ for all then $\mathrm{P} \in \mathrm{H}_{1} \cup \mathrm{H}_{2}$, otherwise $\mathrm{P} \in \mathrm{H}_{4}$.

Remark. By the Sturm separation theorem, $\alpha_{1}, \alpha_{2}$ either both have zeroes or both have none.

Theorem 4.3. If $\mathrm{P} \in \mathrm{H}_{3}$, then $\mathrm{P}^{*}$ has a base of the form

$$
\left(\alpha_{1}(t) e^{\lambda t}, \alpha_{2}(t) e^{\lambda t}\right)
$$

where $\lambda \in \mathbb{R}$ and $\alpha_{1}, \alpha_{2}$ are real-valued $\omega$-periodic functions. 
Proof. If $\mathrm{P} \in \mathrm{H}_{3}$, then a base of $\mathrm{P}^{*}$ has one of the following two forms:

$$
\begin{gathered}
\left(\alpha_{1}(t) e^{\lambda t}, \alpha_{2}(t) e^{\lambda t}\right) \\
\left(\alpha_{1}(t) e^{\lambda t},\left(t \alpha_{1}(t)+\alpha_{2}(t) e^{\lambda t}\right),\right.
\end{gathered}
$$

where $\lambda \in \mathbb{R}$ and $\alpha_{1}, \alpha_{2}$ are real-valued $\omega$-periodic functions. It can be checked that if a base of $P^{*}$ has the form (4.4), then

$$
-\frac{c_{1}\left(\dot{\alpha}_{1}+\lambda \alpha_{1}\right)+c_{2}\left(\left(\dot{\alpha}_{1}+\lambda \alpha_{1}\right) t+\dot{\alpha}_{2}+\lambda \alpha_{2}+\alpha_{1}\right)}{c_{1} \alpha_{1}+c_{2}\left(\alpha_{1} t+\alpha_{2}\right)}
$$

is a solution of $\mathbf{P}$ where $c_{1}, c_{2} \in \mathbb{C}$. Hence $\mathbf{P}$ has at most one period solution, namely

$$
-\frac{\dot{\alpha}_{1}+\lambda \alpha_{1}}{\alpha_{1}}
$$

provided $\alpha_{1}(t) \neq 0$ for all $t$, and the lemma is proved.

Remark. We saw in Lemma 3.5 that if $\mathrm{P} \in H_{11}$, then $\mathrm{P}^{*}$ has Floquet base of the form (4.4). If $\mathrm{P}^{*}$ has this form of base, either $\mathrm{P} \in H_{11}$ or $\mathrm{P} \in H_{4}$. We saw that $H_{11}$ is the boundary "between" $H_{1}$ and $H_{2}$ in the sense that $H_{11}=\bar{H}_{1} \cap \bar{H}_{2}$. We now show that $H_{3}$ is a part of the boundary of $\mathrm{H}_{4}$.

Theorem 4.4. If $\mathrm{P} \in \mathrm{H}_{3}$, then

(i) $\mathrm{P}$ is an accumulation point of $\mathrm{H}_{3}$,

(ii) $\mathrm{P}$ is an accumulation point of $\mathrm{H}_{4}$.

Proof. Since $\mathrm{P} \in H_{3}$, by Theorem 4.3, $\mathrm{P}^{*}$ has a base of the form $\left(\alpha_{1}(t) e^{\lambda t}, \alpha_{2}(t) e^{\lambda(t)}\right)$. Formulae (1.3) and (1.4) give us

$$
p=\frac{\alpha_{2}\left(\ddot{\alpha}_{1}+2 \lambda \dot{\alpha}_{1}+\lambda^{2} \alpha_{1}\right)-\alpha_{1}\left(\ddot{\alpha}_{2}+2 \lambda \dot{\alpha}_{2}+\lambda^{2} \alpha_{2}\right)}{\alpha_{2}\left(\dot{\alpha}_{1}+\lambda \alpha_{1}\right)-\alpha_{1}\left(\dot{\alpha}_{2}+\lambda \alpha_{2}\right)}
$$

and

$$
r=\frac{\left(\ddot{\alpha}_{2}+2 \lambda \dot{\alpha}_{1}+\lambda^{2} \alpha_{1}\right)\left(\dot{\alpha}_{2}+\lambda \alpha_{2}\right)-\left(\ddot{\alpha}_{2}+2 \lambda \dot{\alpha}_{2}+\lambda^{2} \alpha_{2}\right)\left(\dot{\alpha}_{1}+\lambda \alpha_{1}\right)}{\alpha_{2}\left(\dot{\alpha}_{1}+\lambda \alpha_{1}\right)-\alpha_{1}\left(\dot{\alpha}_{2}+\lambda \alpha_{2}\right)}
$$

where $P=(p, r)$ and $\alpha_{2}(t)\left(\dot{\alpha}_{1}(t)+\lambda \alpha_{1}(t)\right)-\alpha_{1}(t)\left(\dot{\alpha}_{2}(t)+\lambda \alpha_{2}(t)\right) \neq 0$ for all $t$ (because $\alpha_{1} e^{\lambda t}$, $\alpha_{2} e^{\lambda t}$ are linearly independent).

To prove (i) consider

$$
u_{n}=\left(\alpha_{1}(t) e^{(\lambda+1 / n) t}, \alpha_{2}(t) e^{(\lambda+1 / n) t}\right), \quad n \in \mathbb{Z}^{+}
$$


It can be checked that $u_{n}$ is a base of $\mathrm{P}_{n}=\left(p_{n}, r_{n}\right)$ where

$$
\begin{aligned}
& p_{n}=\frac{\alpha_{2}\left(\ddot{\alpha}_{1}+2(\lambda+1 / n) \dot{\alpha}_{1}+(\lambda+1 / n)^{2} \alpha_{1}\right)-\alpha_{1}\left(\ddot{\alpha}_{2}+2(\lambda+1 / n) \dot{\alpha}_{2}+(\lambda+1 / n)^{2} \alpha_{2}\right.}{\alpha_{2}\left(\dot{\alpha}_{1}+(\lambda+1 / n) \alpha_{1}\right)-\alpha_{1}\left(\dot{\alpha}_{2}+(\lambda+1 / n) \alpha_{2}\right)} \\
& r_{n}= \\
& \frac{\left(\ddot{\alpha}_{2}+2(\lambda+1 / n) \dot{\alpha}_{1}+(\lambda+1 / n)^{2} \alpha_{1}\right)\left(\dot{\alpha}_{2}+(\lambda+1 / n) \alpha_{2}\right)-\left(\ddot{\alpha}_{2}+2(\lambda+1 / n) \dot{\alpha}_{2}+(\lambda+1 / n)^{2} \alpha_{2}\right)\left(\dot{\alpha}_{1}+(\lambda+1 / n) \alpha_{1}\right)}{\alpha_{2}\left(\dot{\alpha}_{1}+(\lambda+1 / n) \alpha_{1}\right)-\alpha_{1}\left(\dot{\alpha}_{2}+(\lambda+1 / n) \alpha_{2}\right)} .
\end{aligned}
$$

Also $\lim _{n \rightarrow \infty} \mathrm{P}_{n}=\mathrm{P}$ and $\mathrm{P}_{n} \in H_{3}$. Hence (i) is proved.

To prove (ii) consider

$$
u_{n}=\left(\alpha_{1}(t) e^{(\lambda+1 / n) t}, \alpha_{2}(t) e^{\lambda t}\right)
$$

It can be checked that $u_{n}$ is a base of $\mathrm{P}^{n}=\left(p_{n}, r_{n}\right)$ where

$$
\begin{gathered}
P_{n}=\frac{\alpha_{2}\left(\ddot{\alpha}_{1}+2(\lambda+1 / n) \dot{\alpha}_{1}+(\lambda+1 / n)^{2} \alpha_{1}\right)-\alpha_{1}\left(\ddot{\alpha}_{2}+2 \dot{\alpha}_{2}+\lambda^{2} \alpha_{2}\right)}{\alpha_{2}\left(\dot{\alpha}_{1}+(\lambda+1 / n) \alpha_{1}\right)-\alpha_{1}\left(\dot{\alpha}_{2}+\lambda \alpha_{2}\right)} \\
r_{n}=\frac{\left(\ddot{\alpha}_{1}+2(\lambda+1 / n) \dot{\alpha}_{1}+(\lambda+1 / n)^{2} \alpha_{1}\right)\left(\dot{\alpha}_{2}+\lambda \alpha_{2}\right)-\left(\ddot{\alpha}_{2}+2 \lambda \dot{\alpha}_{2}+\lambda^{2} \alpha_{2}\right)\left(\dot{\alpha}_{1}+(\lambda+1 / n) \alpha_{1}\right)}{\alpha_{2}\left(\alpha_{1}^{2}+(\lambda+1 / n) \alpha_{1}\right)-\alpha_{1}\left(\dot{\alpha}_{2}+\lambda_{2}\right)}
\end{gathered}
$$

Now $\lim _{n \rightarrow \infty} \mathrm{P}_{n}=\mathrm{P}$. Since $\mathrm{P} \in H_{3}$, we know that $\alpha_{1}$ must have a zero, otherwise $P$ has a real $\omega$-periodic solution, namely $-\dot{\alpha}_{1}+\lambda \alpha_{1} / \alpha_{1}$. Hence by Lemma $4.2 P_{n} \in H_{4}$ and the theorem is proved.

Remark. Theorem 4.4(i) shows that $H_{3}$ has no isolated member.

Theorem 4.5. $H_{3}=\bar{H}_{4} \backslash H_{4}$.

Proof. Suppose that $\mathrm{P} \in H_{3}$. Then by Theorem 4.4(ii) $\mathrm{P} \in \bar{H}_{4} \backslash H_{4}$. Hence $H_{3} \subset H_{4} \backslash H_{4}$.

Now if $\mathrm{P} \in \bar{H}_{4} \backslash H_{4}$, then either $\mathrm{P} \in H_{1} \cup H_{2}$ or $\mathrm{P} \in H_{3}$. Since $H_{1} \cup H_{2}$ is open, $\mathrm{P} \in H_{3}$ and the theorem is proved.

Remark. Suppose that $\mathrm{P}^{*}$ has a base of the form $\left(\alpha_{1} e^{\lambda t},\left(t \alpha_{1}+\alpha_{2}\right) e^{\lambda t}\right)$ and $\alpha_{1}$ has a zero. Then $\mathrm{P} \varepsilon \mathrm{H}_{4}$. By using the same method used in the proof of Theorem 4.4 we can show that $\mathrm{P} \in \bar{H}_{2}$. Therefore $\mathrm{H}_{3} \neq \partial \mathrm{H}_{4}$.

\section{REFERENCES}

1. F. A. Connington, and N. Levinson, Theory of Ordinary Differential Equations (McGrawIlill. Vill Yurh. 1955). 
2. N. G. Llovd, The number of periodic solutions of the equations $\dot{Z}=z^{N}+p_{1}(t) z^{N}-1+\cdots$ $+p_{n}(t)$, Proc. London Math Soc. 27 (1973), 667-700.

3. N. G. Lloyd, On analytic differential equations, Proc. London Math. Soc. (3) 30, (1975), 430-44.

4. N. G. LloYd, On a class of differential equations of Riccati type, J. London Math. Soc. (2) 10 (1975), 1-10.

5. N. G. Lloyd, Degree Theory (Cambridge University Press, 1978).

Department of Mathematics

UNIVERSITY OF BIRMINGHAM

P.O. Box 363, Edgbaston

Birmingham. B15 2TT 